First Study of Metal Hybrids of The Boronic Acids: Second-Sphere Coordination Networks in the Structures of 4-Carboxyphenylboronic Acid With Some Transition Metals

Nanappan SeethaLekshmi and Venkateswara Rao Pedireddi ${ }^{\star}$

Solid State \& Supramolecular Structural Chemistry Lab, Division of Organic Chemistry, National Chemical Laboratory, Dr. Homi Bhabha Road, Pune 411 008, India.

Email:vr.pedireddi@ncl.res.in

\title{
Index:
}

\begin{tabular}{|l|l|l|}
\hline Content & Description & Page No. \\
\hline Complex 1a & ORTEP drawing & S2 \\
\hline & Experimental information & S2 \\
\hline & Crystallographic information Tables & S3-S12 \\
\hline Complex 1b & ORTEP drawing & S13 \\
\hline & Experimental information & S13 \\
\hline & Crystal packing figures & S14 \\
\hline & Crystallographic information Tables & S15-S24 \\
\hline Complex 1c & ORTEP drawing & S25 \\
\hline & Experimental information & S25 \\
\hline & Crystal packing figures & S26 \\
\hline & Crystallographic information Tables & S27-S35 \\
\hline Complex 1d & ORTEP drawing & S36 \\
\hline & Experimental information & S36 \\
\hline & Crystallographic information Tables & S37-S60 \\
\hline Hydrogen bonds & Comples 1a - 1d & S61 \\
\hline
\end{tabular}




\section{Complex 1a:}
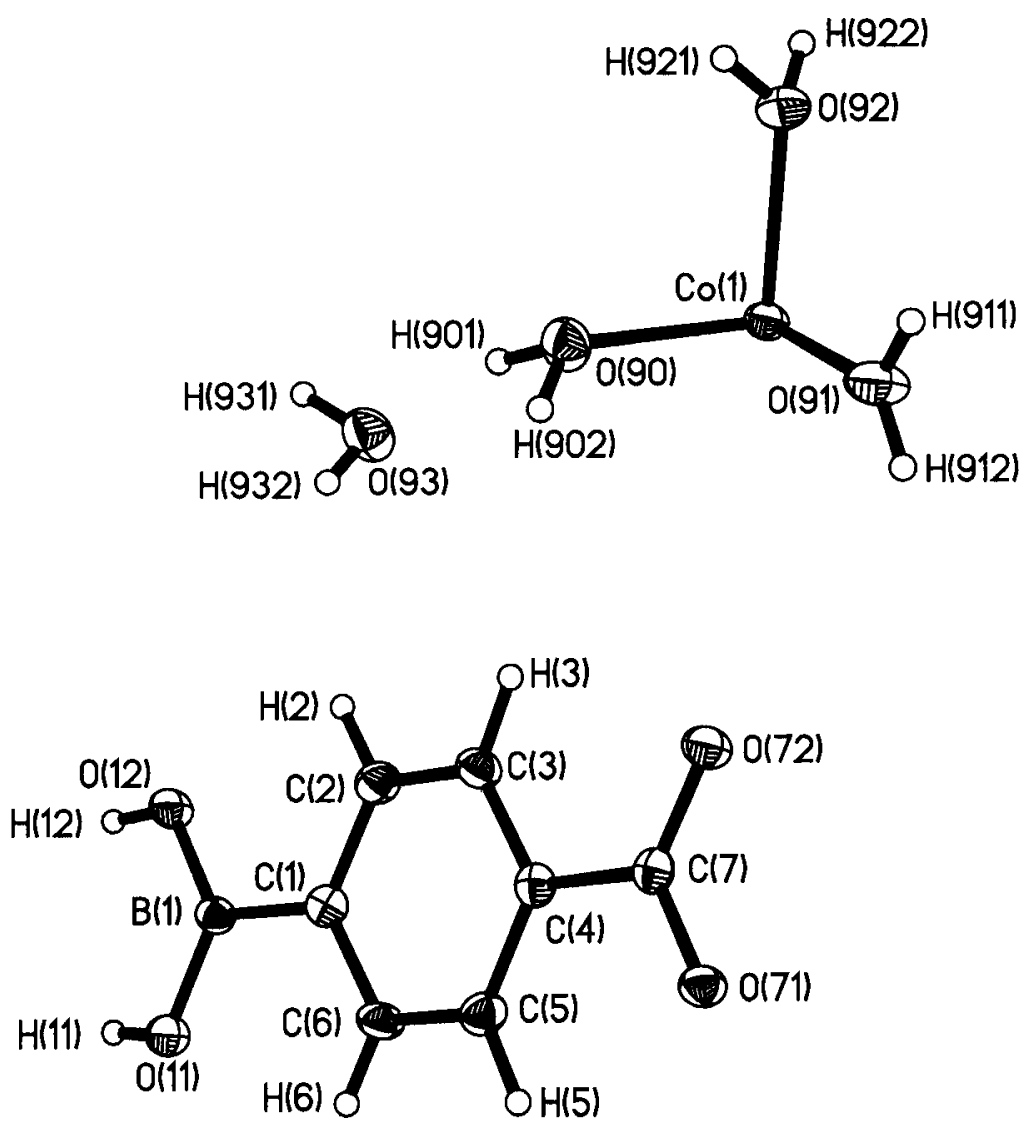

H(941) $O H(942)$

\section{Experimental Information}

A methanolic solution $(5 \mathrm{~mL})$ of sodium salt of 4-carboxyphenylboronic acid $(0.187 \mathrm{~g}, 1 \mathrm{mmol})$ was added, to a hot aqueous solution of $\mathrm{Co}\left(\mathrm{NO}_{3}\right)_{2} .6 \mathrm{H}_{2} \mathrm{O}(0.291 \mathrm{~g}, 1 \mathrm{mmol})$, with constant stirring over a period of five minutes. The reaction mixture was warmed for a while and the resulting pale pink colored solution was filtered and kept for slow evaporation at ambient conditions. Single crystals, (pink colored) suitable for X-ray diffraction studies were obtained after one week. (Yield: 64\%). 


\section{Crystallographic Information}

Table 1. Crystal data and structure refinement for $\mathbf{1} \mathbf{a}$.

Identification code

Empirical formula

Formula weight

Temperature

Wavelength

Crystal system

Space group

Unit cell dimensions

Volume

Z

Density (calculated)

Absorption coefficient

$F(000)$

Crystal size

Theta range for data collection

Index ranges

Reflections collected

Independent reflections

Completeness to theta $=28.28^{\circ}$

Absorption correction

Refinement method

Data / restraints / parameters

Goodness-of-fit on $\mathrm{F}^{2}$

Final $R$ indices $[\mathrm{l}>2 \operatorname{sigma}(\mathrm{l})]$

$\mathrm{R}$ indices (all data)

Largest diff. peak and hole
$1 \mathrm{a}$

$\mathrm{C}_{14} \mathrm{H}_{32} \mathrm{~B}_{2} \mathrm{O}_{18} \mathrm{Co}$

568.95

133(2) K

$0.71073 \AA$

Triclinic

$\mathrm{P}_{\overline{1}}$

$\mathrm{a}=6.776(3) \AA \quad \alpha=98.19(7)^{\circ}$.

$\mathrm{b}=9.592(4) \AA \quad \beta=91.66(8)^{\circ}$.

$\mathrm{c}=9.786(5) \AA \quad \gamma=108.35(7)^{\circ}$.

$595.7(5) \AA^{3}$

1

$1.586 \mathrm{Mg} / \mathrm{m}^{3}$

$0.804 \mathrm{~mm}^{-1}$

297

$0.29 \times 0.25 \times 0.17 \mathrm{~mm}^{3}$

2.11 to $28.28^{\circ}$.

$-8 \leq h \geq 8,-12 \leq k \geq 12,-12 \leq 1 \geq 13$

6817

$2699[\mathrm{R}($ int $)=0.0263]$

$92.0 \%$

Empirical

Full-matrix least-squares on $\mathrm{F}^{2}$

$2699 / 0 / 224$

0.963

$\mathrm{R} 1=0.0414, \mathrm{wR} 2=0.0989$

$\mathrm{R} 1=0.0495, \mathrm{wR} 2=0.1004$

1.382 and -0.293 e. $\AA^{-3}$ 
Table 2. Atomic coordinates ( $\left.\times 10^{4}\right)$ and equivalent isotropic displacement parameters $\left(\AA^{2} \times 10^{3}\right)$ for $1 \mathbf{a} . \mathrm{U}(\mathrm{eq})$ is defined as one third of the trace of the orthogonalized $\mathrm{U}^{\mathrm{ij}}$ tensor.

\begin{tabular}{lrrrr}
\hline & $\mathrm{x}$ & $\mathrm{y}$ & $\mathrm{z}$ & $\mathrm{U}(\mathrm{eq})$ \\
\hline $\mathrm{Co}(1)$ & \multicolumn{1}{c}{} & & & \\
$\mathrm{O}(72)$ & 0 & 0 & 0 & $16(1)$ \\
$\mathrm{O}(12)$ & $2447(3)$ & $4501(2)$ & $154(2)$ & $20(1)$ \\
$\mathrm{O}(71)$ & $3353(3)$ & $6934(2)$ & $-6819(2)$ & $19(1)$ \\
$\mathrm{O}(92)$ & $3633(2)$ & $6984(2)$ & $497(2)$ & $17(1)$ \\
$\mathrm{O}(91)$ & $404(3)$ & $-1848(2)$ & $600(2)$ & $21(1)$ \\
$\mathrm{O}(11)$ & $2882(3)$ & $487(2)$ & $-852(2)$ & $23(1)$ \\
$\mathrm{O}(90)$ & $2241(3)$ & $4294(2)$ & $-7180(2)$ & $20(1)$ \\
$\mathrm{C}(7)$ & $1473(3)$ & $1173(2)$ & $1942(2)$ & $26(1)$ \\
$\mathrm{C}(5)$ & $3007(3)$ & $5720(3)$ & $-304(2)$ & $15(1)$ \\
$\mathrm{C}(3)$ & $3368(4)$ & $7021(3)$ & $-2389(2)$ & $18(1)$ \\
$\mathrm{C}(1)$ & $2361(4)$ & $4363(3)$ & $-2751(2)$ & $18(1)$ \\
$\mathrm{C}(6)$ & $2777(3)$ & $5649(3)$ & $-4728(2)$ & $16(1)$ \\
$\mathrm{C}(4)$ & $3309(4)$ & $6989(3)$ & $-3809(2)$ & $19(1)$ \\
$\mathrm{B}(1)$ & $2912(3)$ & $5711(2)$ & $-1844(2)$ & $15(1)$ \\
$\mathrm{C}(2)$ & $2779(4)$ & $5608(3)$ & $-6329(3)$ & $15(1)$ \\
$\mathrm{O}(94)$ & $2302(4)$ & $4349(3)$ & $-4159(2)$ & $19(1)$ \\
$\mathrm{O}(93)$ & $1566(4)$ & $9233(2)$ & $-6166(2)$ & $25(1)$ \\
\hline
\end{tabular}

Table 3. Bond lengths $[\AA]$ and angles $\left[{ }^{\circ}\right]$ for $\mathbf{1} \mathbf{a}$.

\begin{tabular}{|c|c|c|c|}
\hline 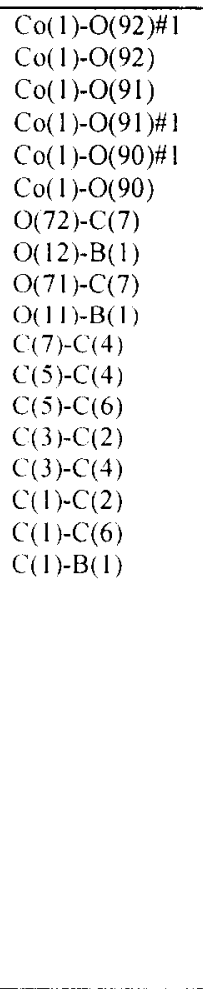 & $\begin{array}{l}2.0384(19) \\
2.0384(19) \\
2.0875(19) \\
2.0875(19) \\
2.109(2) \\
2.109(2) \\
1.261(3) \\
1.368(3) \\
1.285(3) \\
1.345(3) \\
1.506(3) \\
1.384(3) \\
1.385(3) \\
1.376(3) \\
1.396(3) \\
1.387(3) \\
1.396(3) \\
1.562(3)\end{array}$ & $\begin{array}{l}\mathrm{O}(92) \# 1-\mathrm{Co}(1)-\mathrm{O}(92) \\
\mathrm{O}(92) \# 1-\mathrm{Co}(1)-\mathrm{O}(91) \\
\mathrm{O}(92)-\mathrm{Co}(1)-\mathrm{O}(91) \\
\mathrm{O}(92) \# 1-\mathrm{Co}(1)-\mathrm{O}(91) \# 1 \\
\mathrm{O}(92)-\mathrm{Co}(1)-\mathrm{O}(91) \# 1 \\
\mathrm{O}(91)-\mathrm{Co}(1)-\mathrm{O}(91) \# 1 \\
\mathrm{O}(92) \# 1-\mathrm{Co}(1)-\mathrm{O}(90) \# 1 \\
\mathrm{O}(92)-\mathrm{Co}(1)-\mathrm{O}(90) \# 1 \\
\mathrm{O}(91)-\mathrm{Co}(1)-\mathrm{O}(90) \# 1 \\
\mathrm{O}(91) \# 1-\mathrm{Co}(1)-\mathrm{O}(90) \# 1 \\
\mathrm{O}(92) \# 1-\mathrm{Co}(1)-\mathrm{O}(90) \\
\mathrm{O}(92)-\mathrm{C}(1)-\mathrm{O}(90) \\
\mathrm{O}(91)-\mathrm{Co}(1)-\mathrm{O}(90) \\
\mathrm{O}(91) \# 1-\mathrm{Co}(1)-\mathrm{O}(90) \\
\mathrm{O}(90) \# 1-\mathrm{Co}(1)-\mathrm{O}(90) \\
\mathrm{O}(72)-\mathrm{C}(7)-\mathrm{O}(71) \\
\mathrm{O}(72)-\mathrm{C}(7)-\mathrm{C}(4) \\
\mathrm{O}(71)-\mathrm{C}(7)-\mathrm{C}(4) \\
\mathrm{C}(4)-\mathrm{C}(5)-\mathrm{C}(6) \\
\mathrm{C}(2)-\mathrm{C}(3)-\mathrm{C}(4) \\
\mathrm{C}(2)-\mathrm{C}(1)-\mathrm{C}(6) \\
\mathrm{C}(2)-\mathrm{C}(1)-\mathrm{B}(1) \\
\mathrm{C}(6)-\mathrm{C}(1)-\mathrm{B}(1) \\
\mathrm{C}(5)-\mathrm{C}(6)-\mathrm{C}(1) \\
\mathrm{C}(5)-\mathrm{C}(4)-\mathrm{C}(3) \\
\mathrm{C}(5)-\mathrm{C}(4)-\mathrm{C}(7) \\
\mathrm{C}(3)-\mathrm{C}(4)-\mathrm{C}(7) \\
\mathrm{O}(11)-\mathrm{B}(1)-\mathrm{O}(12) \\
\mathrm{O}(11)-\mathrm{B}(1)-\mathrm{C}(1) \\
\mathrm{O}(12)-\mathrm{B}(1)-\mathrm{C}(1) \\
\mathrm{C}(3)-\mathrm{C}(2)-\mathrm{C}(1)\end{array}$ & $\begin{array}{c}180.00(14) \\
89.87(8) \\
90.13(8) \\
90.13(8) \\
89.87(8) \\
180.00(10) \\
87.22(8) \\
92.78(8) \\
89.69(8) \\
90.31(8) \\
92.78(8) \\
87.22(8) \\
90.31(8) \\
89.69(8) \\
180.00(12) \\
122.5(2) \\
119.4(2) \\
118.1(2) \\
120.3(2) \\
120.2(2) \\
117.2(2) \\
121.0(2) \\
121.7(2) \\
121.5(2) \\
118.8(2) \\
121.3(2) \\
119.9(2) \\
122.0(2) \\
119.9(2) \\
118.1(2) \\
122.0(2)\end{array}$ \\
\hline
\end{tabular}


Table 4. Anisotropic displacement parameters $\left(\AA^{2} \times 10^{3}\right)$ for $1 \mathbf{a}$. The anisotropic displacement factor exponent takes the form: $-2 \pi^{2}\left[h^{2} a^{* 2} U^{11}+\ldots+2 h k a^{*} b^{*} U^{12}\right]$

\begin{tabular}{lcccccc}
\hline & $\mathrm{U}^{11}$ & $\mathrm{U}^{22}$ & $\mathrm{U}^{33}$ & $\mathrm{U}^{23}$ & $\mathrm{U}^{13}$ & $\mathrm{U}^{12}$ \\
\hline $\mathrm{Co}(1)$ & $19(1)$ & $12(1)$ & $16(1)$ & $3(1)$ & $1(1)$ & $3(1)$ \\
$\mathrm{O}(72)$ & $31(1)$ & $15(1)$ & $12(1)$ & $2(1)$ & $1(1)$ & $3(1)$ \\
$\mathrm{O}(12)$ & $28(1)$ & $16(1)$ & $10(1)$ & $2(1)$ & $2(1)$ & $5(1)$ \\
$\mathrm{O}(71)$ & $22(1)$ & $14(1)$ & $14(1)$ & $2(1)$ & $0(1)$ & $4(1)$ \\
$\mathrm{O}(92)$ & $22(1)$ & $14(1)$ & $24(1)$ & $2(1)$ & $1(1)$ & $4(1)$ \\
$\mathrm{O}(91)$ & $21(1)$ & $22(1)$ & $22(1)$ & $2(1)$ & $2(1)$ & $2(1)$ \\
$\mathrm{O}(11)$ & $30(1)$ & $17(1)$ & $11(1)$ & $5(1)$ & $2(1)$ & $5(1)$ \\
$\mathrm{O}(90)$ & $39(1)$ & $15(1)$ & $21(1)$ & $4(1)$ & $-5(1)$ & $4(1)$ \\
$\mathrm{C}(7)$ & $17(1)$ & $17(1)$ & $12(1)$ & $2(1)$ & $1(1)$ & $5(1)$ \\
$\mathrm{C}(5)$ & $24(1)$ & $16(1)$ & $14(1)$ & $1(1)$ & $-1(1)$ & $6(1)$ \\
$\mathrm{C}(3)$ & $24(1)$ & $14(1)$ & $16(1)$ & $4(1)$ & $2(1)$ & $4(1)$ \\
$\mathrm{C}(1)$ & $16(1)$ & $19(1)$ & $12(1)$ & $3(1)$ & $0(1)$ & $5(1)$ \\
$\mathrm{C}(6)$ & $27(1)$ & $13(1)$ & $18(1)$ & $6(1)$ & $2(1)$ & $6(1)$ \\
$\mathrm{C}(4)$ & $15(1)$ & $18(1)$ & $12(1)$ & $3(1)$ & $1(1)$ & $5(1)$ \\
$\mathrm{B}(1)$ & $16(1)$ & $15(1)$ & $14(1)$ & $3(1)$ & $0(1)$ & $4(1)$ \\
$\mathrm{C}(2)$ & $23(1)$ & $15(1)$ & $16(1)$ & $0(1)$ & $0(1)$ & $4(1)$ \\
$\mathrm{O}(94)$ & $33(1)$ & $21(1)$ & $22(1)$ & $1(1)$ & $-1(1)$ & $10(1)$ \\
$\mathrm{O}(93)$ & $27(1)$ & $23(1)$ & $20(1)$ & $1(1)$ & $2(1)$ & $-1(1)$ \\
\hline
\end{tabular}

Table 5. Hydrogen coordinates $\left(\times 10^{4}\right)$ and isotropic displacement parameters $\left(\AA^{2} \times 10^{3}\right)$ for $\mathbf{1 a}$.

\begin{tabular}{|c|c|c|c|c|}
\hline & $\mathrm{x}$ & $y$ & $\mathrm{z}$ & $\mathrm{U}(\mathrm{eq})$ \\
\hline $\mathrm{H}(3)$ & $2000(40)$ & $3430(30)$ & $-2410(30)$ & $21(7)$ \\
\hline $\mathrm{H}(922)$ & $-490(50)$ & $-2640(30)$ & $430(30)$ & $22(8)$ \\
\hline$H(5)$ & $3780(40)$ & $7970(30)$ & $-1800(30)$ & $24(7)$ \\
\hline $\mathrm{H}(6)$ & $3640(40)$ & $7850(30)$ & $-4180(30)$ & $19(7)$ \\
\hline $\mathrm{H}(942)$ & $330(60)$ & $8990(40)$ & $-6250(40)$ & $44(11)$ \\
\hline $\mathrm{H}(2)$ & $1930(40)$ & $3440(30)$ & $-4770(30)$ & $25(7)$ \\
\hline $\mathrm{H}(941)$ & $2040(50)$ & $8650(40)$ & $-6340(40)$ & $46(12)$ \\
\hline $\mathrm{H}(932)$ & $3550(50)$ & $1480(40)$ & $-3650(30)$ & $26(9)$ \\
\hline $\mathrm{H}(921)$ & $1500(60)$ & $-2110(40)$ & $500(40)$ & $48(10)$ \\
\hline $\mathrm{H}(11)$ & $2290(50)$ & $4340(30)$ & $-8040(30)$ & $40(9)$ \\
\hline $\mathrm{H}(912)$ & $3900(60)$ & $1300(40)$ & $-630(40)$ & $59(11)$ \\
\hline $\mathrm{H}(902)$ & $1800(50)$ & $2090(40)$ & $2300(30)$ & $44(10)$ \\
\hline $\mathrm{H}(901)$ & $1760(50)$ & $730(30)$ & $2490(30)$ & $29(9)$ \\
\hline $\mathrm{H}(911)$ & $2740(50)$ & $460(40)$ & $-1690(40)$ & $46(10)$ \\
\hline $\mathrm{H}(12)$ & $3330(50)$ & $6840(40)$ & $-7680(40)$ & $55(11)$ \\
\hline $\mathrm{H}(931)$ & $2420(80)$ & $270(60)$ & $-4490(60)$ & $110(17)$ \\
\hline
\end{tabular}




\section{Complex 1b}
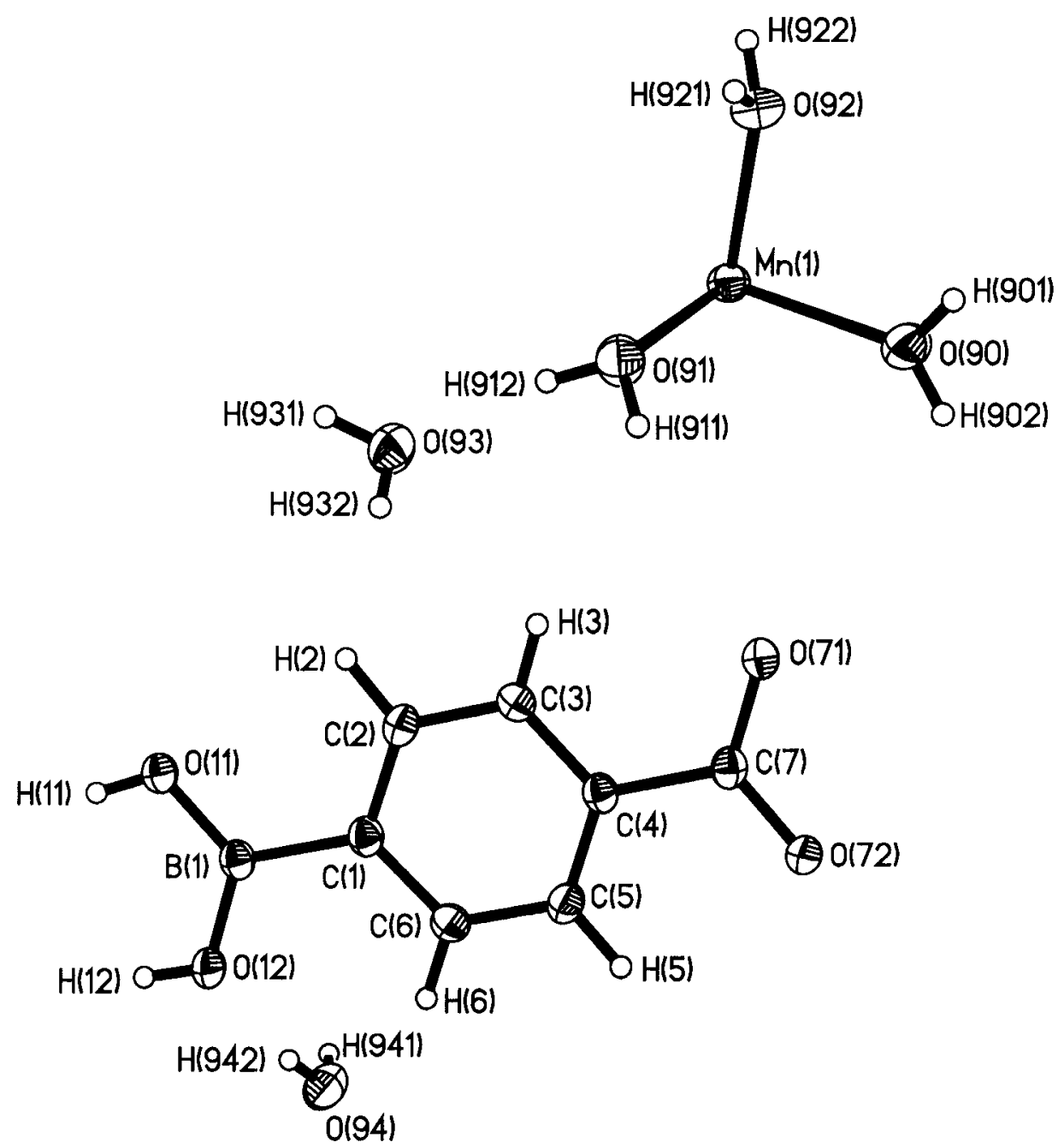

\section{Experimental Information:}

To a hot aqueous solution of $\mathrm{Mn}\left(\mathrm{NO}_{3}\right)_{2}$. $6 \mathrm{H}_{2} \mathrm{O}(0.287 \mathrm{~g}, 1 \mathrm{mmol})$, was added a methanolic solution $(5 \mathrm{~mL})$ of sodium salt of 4-carboxyphenylboronic acid $(0.187 \mathrm{~g}, 1 \mathrm{mmol})$, with constant stirring over a period of five minutes. The reaction mixture was warmed for a while and the resulting colorless solution was filtered and kept for slow evaporation at room temperature. Pale yellow single crystals suitable for x-ray diffraction studies were obtained after 4 days. (Yield: $48 \%$ ). 

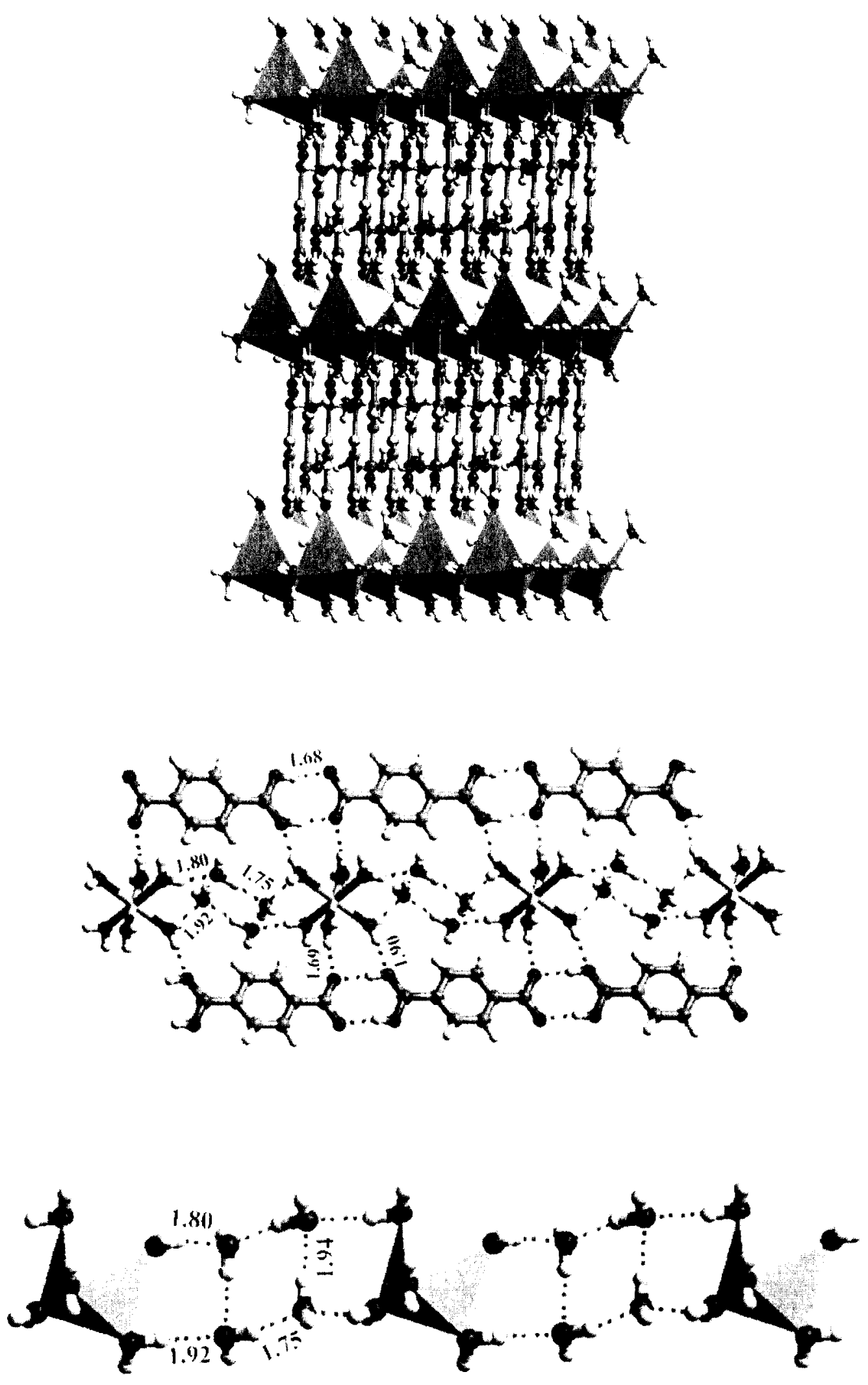


\section{Crystallographic information:}

\begin{tabular}{|c|c|c|}
\hline Identification code & \multicolumn{2}{|l|}{ lb } \\
\hline Empirical formula & \multicolumn{2}{|c|}{$\mathrm{C}_{14} \mathrm{H}_{32} \mathrm{~B}_{2} \mathrm{O}_{18} \mathrm{Mn}$} \\
\hline Formula weight & \multicolumn{2}{|l|}{564.96} \\
\hline Temperature & \multicolumn{2}{|l|}{$133(2) \mathrm{K}$} \\
\hline Wavelength & \multicolumn{2}{|l|}{$0.71073 \AA$} \\
\hline Crystal system & \multicolumn{2}{|l|}{ Triclinic } \\
\hline Space group & \multicolumn{2}{|l|}{$P \overline{1}$} \\
\hline \multirow[t]{3}{*}{ Unit cell dimensions } & $a=6.862(2) \AA$ & $\alpha=98.33(5)^{\circ}$ \\
\hline & $b=9.705(3) \AA$ & $\beta=92.2 \mathrm{l}(5)^{\circ}$ \\
\hline & $c=9.806(3) \AA$ & $\gamma=108.22(5)^{\circ}$. \\
\hline Volume & \multicolumn{2}{|l|}{$611.3(3) \AA^{3}$} \\
\hline Z & \multicolumn{2}{|l|}{1} \\
\hline Density (calculated) & \multicolumn{2}{|l|}{$1.535 \mathrm{Mg} / \mathrm{m}^{3}$} \\
\hline Absorption coefficient & \multicolumn{2}{|l|}{$0.621 \mathrm{~mm}^{-1}$} \\
\hline $\mathrm{F}(000)$ & \multicolumn{2}{|l|}{295} \\
\hline Crystal size & \multicolumn{2}{|c|}{$0.29 \times 0.22 \times 0.19 \mathrm{~mm}^{3}$} \\
\hline Theta range for data collection & \multicolumn{2}{|l|}{2.11 to $28.35^{\circ}$. } \\
\hline Index ranges & \multicolumn{2}{|c|}{$-9 \leq h \geq 9,-12 \leq k \geq 12,-12 \leq 1 \geq 13$} \\
\hline Reflections collected & \multicolumn{2}{|l|}{7016} \\
\hline Independent reflections & \multicolumn{2}{|c|}{$2776[\mathrm{R}(\mathrm{int})=0.0178]$} \\
\hline Completeness to theta $=28.35^{\circ}$ & \multicolumn{2}{|l|}{$91.2 \%$} \\
\hline Absorption correction & \multicolumn{2}{|l|}{ Empirical } \\
\hline Refinement method & \multicolumn{2}{|c|}{ Full-matrix least-squares on $\mathrm{F}^{2}$} \\
\hline Data / restraints / parameters & \multicolumn{2}{|l|}{$2776 / 0 / 224$} \\
\hline Goodness-of-fit on $\mathrm{F}^{2}$ & \multicolumn{2}{|l|}{1.022} \\
\hline Final $R$ indices $[I>2 \operatorname{sigma}(I)]$ & \multicolumn{2}{|c|}{$\mathrm{R} 1=0.0340, w \mathrm{R} 2=0.0879$} \\
\hline $\mathrm{R}$ indices (all data) & \multicolumn{2}{|c|}{$\mathrm{R} 1=0.0380, w \mathrm{R} 2=0.0892$} \\
\hline Largest diff. peak and hole & \multicolumn{2}{|c|}{0.699 and $-0.223 \mathrm{e} . \AA^{-3}$} \\
\hline
\end{tabular}


Table 2. Atomic coordinates $\left(\times 10^{4}\right)$ and equivalent isotropic displacement parameters $\left(\AA^{2} \times 10^{3}\right)$ for $\mathbf{1 b}$. $U(e q)$ is defined as one third of the trace of the orthogonalized $U^{i j}$ tensor.

\begin{tabular}{|c|c|c|c|c|}
\hline & $x$ & $y$ & $\mathrm{z}$ & $\mathrm{U}(\mathrm{eq})$ \\
\hline $\operatorname{Mn}(1)$ & 0 & 0 & 0 & $21(1)$ \\
\hline$O(90)$ & $1419(2)$ & $1201(2)$ & $2049(1)$ & $34(1)$ \\
\hline$O(91)$ & $2970(2)$ & $537(1)$ & $-875(1)$ & $29(1)$ \\
\hline$O(92)$ & $422(2)$ & $-1911(1)$ & $616(1)$ & $26(1)$ \\
\hline$O(93)$ & $2667(2)$ & $927(2)$ & $-3616(1)$ & $33(1)$ \\
\hline$O(94)$ & $1544(3)$ & $9205(1)$ & $-6140(1)$ & $33(1)$ \\
\hline$C(1)$ & $2782(2)$ & $5646(2)$ & $-4717(1)$ & $20(1)$ \\
\hline$C(2)$ & $2331(2)$ & $4356(2)$ & $-4157(2)$ & $23(1)$ \\
\hline C(3) & $2405(2)$ & $4376(2)$ & $-2742(2)$ & $22(1)$ \\
\hline$C(4)$ & $2925(2)$ & $5700(2)$ & $-1836(1)$ & $19(1)$ \\
\hline$C(5)$ & $3354(2)$ & $7001(2)$ & $-2368(2)$ & $23(1)$ \\
\hline$C(6)$ & $3287(3)$ & $6968(2)$ & $-3789(2)$ & $24(1)$ \\
\hline$C(7)$ & $3013(2)$ & $5711(2)$ & $-295(1)$ & $18(1)$ \\
\hline$B(1)$ & $2776(2)$ & $5607(2)$ & $-6321(2)$ & $19(1)$ \\
\hline$O(11)$ & $2258(2)$ & $4302(1)$ & $-7176(1)$ & $24(1)$ \\
\hline$O(12)$ & $3334(2)$ & $6916(1)$ & $-6804(1)$ & $24(1)$ \\
\hline$O(71)$ & $2472(2)$ & $4506(1)$ & 149(1) & $25(1)$ \\
\hline$O(72)$ & $3618(2)$ & $6952(1)$ & $508(1)$ & $21(1)$ \\
\hline
\end{tabular}

Table 3. Bond lengths $[\AA]$ and angles $\left[{ }^{\circ}\right]$ for $\mathbf{1 b}$.

\begin{tabular}{|c|c|c|c|}
\hline $\mathrm{Mn}(1)-\mathrm{O}(92) \# 1$ & $2.1300(13)$ & $\mathrm{O}(92) \# 1-\mathrm{Mn}(1)-\mathrm{O}(92)$ & $180.00(6)$ \\
\hline $\mathrm{Mn}(1)-\mathrm{O}(92)$ & $2.1300(13)$ & $\mathrm{O}(92) \# 1-\mathrm{Mn}(1)-\mathrm{O}(91) \# 1$ & $90.91(5)$ \\
\hline $\mathrm{Mn}(1)-\mathrm{O}(91) \# 1$ & $2.1821(13)$ & $\mathrm{O}(92)-\mathrm{Mn}(1)-\mathrm{O}(91) \# 1$ & $89.09(5)$ \\
\hline $\mathrm{Mn}(1)-\mathrm{O}(91)$ & $2.1821(13)$ & $\mathrm{O}(92) \# 1-\mathrm{Mn}(1)-\mathrm{O}(91)$ & $89.09(5)$ \\
\hline $\operatorname{Mn}(1)-O(90)$ & $2.1917(14)$ & $\mathrm{O}(92)-\mathrm{Mn}(1)-\mathrm{O}(91)$ & $90.91(5)$ \\
\hline $\mathrm{Mn}(1)-\mathrm{O}(90) \# 1$ & $2.1917(14)$ & $\mathrm{O}(91) \# 1-\mathrm{Mn}(1)-\mathrm{O}(91)$ & $180.00(6)$ \\
\hline$C(1)-C(2)$ & $1.391(2)$ & $\mathrm{O}(92) \# 1-\mathrm{Mn}(1)-\mathrm{O}(90)$ & $92.28(5)$ \\
\hline$C(1)-C(6)$ & $1.398(2)$ & $O(92)-\mathrm{Mn}(1)-\mathrm{O}(90)$ & $87.72(5)$ \\
\hline$C(1)-B(1)$ & $1.568(2)$ & $O(91) \# 1-M n(1)-O(90)$ & $88.01(5)$ \\
\hline$C(2)-C(3)$ & $1.383(2)$ & $\mathrm{O}(91)-\mathrm{Mn}(1)-\mathrm{O}(90)$ & $91.99(5)$ \\
\hline$C(3)-C(4)$ & $1.389(2)$ & $\mathrm{O}(92) \# 1-\mathrm{Mn}(1)-\mathrm{O}(90) \# 1$ & $87.72(5)$ \\
\hline$C(4)-C(5)$ & $1.387(2)$ & $O(92)-\mathrm{Mn}(1)-O(90) \# 1$ & $92.28(5)$ \\
\hline$C(4)-C(7)$ & $1.509(2)$ & $\mathrm{O}(91) \# 1-\mathrm{Mn}(1)-\mathrm{O}(90) \# 1$ & $91.99(5)$ \\
\hline$C(5)-C(6)$ & $1.387(2)$ & $O(91)-\mathrm{Mn}(1)-O(90) \# 1$ & $88.01(5)$ \\
\hline$C(7)-O(71)$ & $1.2585(17)$ & $O(90)-\mathrm{Mn}(1)-\mathrm{O}(90) \# \mathrm{I}$ & $180.00(8)$ \\
\hline$C(7)-O(72)$ & $1.2765(18)$ & $C(2)-C(1)-C(6)$ & $117.17(13)$ \\
\hline $\mathrm{B}(1)-\mathrm{O}(11)$ & $1.349(2)$ & $\mathrm{C}(2)-\mathrm{C}(1)-\mathrm{B}(1)$ & $120.92(13)$ \\
\hline \multirow[t]{14}{*}{$B(1)-O(12)$} & $1.367(2)$ & $C(6)-C(1)-B(1)$ & $121.89(13)$ \\
\hline & & $C(3)-C(2)-C(1)$ & $121.63(14)$ \\
\hline & & $C(2)-C(3)-C(4)$ & $120.40(14)$ \\
\hline & & $C(5)-C(4)-C(3)$ & $119.08(13)$ \\
\hline & & $C(5)-C(4)-C(7)$ & $120.92(13)$ \\
\hline & & $C(3)-C(4)-C(7)$ & $120.00(13)$ \\
\hline & & $C(4)-C(5)-C(6)$ & $120.03(14)$ \\
\hline & & $C(5)-C(6)-C(1)$ & $121.69(14)$ \\
\hline & & $\mathrm{O}(71)-\mathrm{C}(7)-\mathrm{O}(72)$ & $122.68(13)$ \\
\hline & & $\mathrm{O}(71)-\mathrm{C}(7)-\mathrm{C}(4)$ & $119.07(13)$ \\
\hline & & $\mathrm{O}(72)-\mathrm{C}(7)-\mathrm{C}(4)$ & $118.24(12)$ \\
\hline & & $\mathrm{O}(11)-\mathrm{B}(1)-\mathrm{O}(12)$ & $122.18(14)$ \\
\hline & & $\mathrm{O}(11)-\mathrm{B}(1)-\mathrm{C}(1)$ & $119.66(14)$ \\
\hline & & $O(12)-B(1)-C(1)$ & $118.15(13)$ \\
\hline
\end{tabular}


Table 4. Anisotropic displacement parameters $\left(\AA^{2} \times 10^{3}\right)$ for $\mathbf{1 b}$. The anisotropic displacement factor exponent takes the form: $-2 \pi^{2}\left[h^{2} a^{* 2} U^{11}+\ldots+2 h k a^{*} b^{*} U^{12}\right]$

\begin{tabular}{lllllll}
\hline & $U^{11}$ & $U^{22}$ & $U^{33}$ & $U^{23}$ & $U^{13}$ & $U^{12}$ \\
\hline$M(1)$ & $22(1)$ & $18(1)$ & $22(1)$ & $4(1)$ & $1(1)$ & $4(1)$ \\
$O(90)$ & $48(1)$ & $23(1)$ & $27(1)$ & $3(1)$ & $-7(1)$ & $6(1)$ \\
$O(91)$ & $24(1)$ & $31(1)$ & $27(1)$ & $3(1)$ & $2(1)$ & $2(1)$ \\
$O(92)$ & $25(1)$ & $19(1)$ & $34(1)$ & $4(1)$ & $1(1)$ & $6(1)$ \\
$O(93)$ & $35(1)$ & $31(1)$ & $26(1)$ & $-2(1)$ & $1(1)$ & $2(1)$ \\
$O(94)$ & $41(1)$ & $28(1)$ & $28(1)$ & $0(1)$ & $-2(1)$ & $13(1)$ \\
$C(1)$ & $20(1)$ & $23(1)$ & $16(1)$ & $3(1)$ & $0(1)$ & $6(1)$ \\
$C(2)$ & $28(1)$ & $22(1)$ & $17(1)$ & $0(1)$ & $1(1)$ & $6(1)$ \\
$C(3)$ & $28(1)$ & $19(1)$ & $19(1)$ & $5(1)$ & $3(1)$ & $6(1)$ \\
$C(4)$ & $18(1)$ & $22(1)$ & $16(1)$ & $4(1)$ & $1(1)$ & $6(1)$ \\
$C(5)$ & $28(1)$ & $21(1)$ & $18(1)$ & $1(1)$ & $0(1)$ & $7(1)$ \\
$C(6)$ & $32(1)$ & $20(1)$ & $20(1)$ & $6(1)$ & $2(1)$ & $8(1)$ \\
$C(7)$ & $16(1)$ & $22(1)$ & $16(1)$ & $2(1)$ & $1(1)$ & $7(1)$ \\
$B(1)$ & $18(1)$ & $23(1)$ & $16(1)$ & $3(1)$ & $1(1)$ & $7(1)$ \\
$O(1)$ & $33(1)$ & $22(1)$ & $15(1)$ & $4(1)$ & $2(1)$ & $6(1)$ \\
$O(12)$ & $33(1)$ & $22(1)$ & $14(1)$ & $2(1)$ & $2(1)$ & $7(1)$ \\
$O(71)$ & $35(1)$ & $21(1)$ & $16(1)$ & $3(1)$ & $1(1)$ & $4(1)$ \\
$O(72)$ & $25(1)$ & $19(1)$ & $16(1)$ & $2(1)$ & $0(1)$ & $5(1)$ \\
\hline
\end{tabular}

Table 5. Hydrogen coordinates $\left(\times 10^{4}\right)$ and isotropic displacement parameters $\left(\AA^{2} \times 10^{3}\right)$ for $\mathbf{1 b}$.

\begin{tabular}{lrrrr}
\hline & $x$ & $y$ & $z$ & U(eq) \\
\hline$H(921)$ & $1400(40)$ & $-2140(20)$ & $510(20)$ & $41(6)$ \\
$H(922)$ & $-550(30)$ & $-2740(20)$ & $470(20)$ & $31(5)$ \\
$H(931)$ & $2490(40)$ & $370(30)$ & $-4330(30)$ & $65(8)$ \\
$H(932)$ & $3540(40)$ & $1550(30)$ & $-3680(20)$ & $45(7)$ \\
$H(941)$ & $440(40)$ & $8940(30)$ & $-6290(30)$ & $54(9)$ \\
$H(942)$ & $2110(40)$ & $8620(30)$ & $-6380(30)$ & $69(9)$ \\
$H(11)$ & $2300(40)$ & $4380(20)$ & $-7990(30)$ & $56(7)$ \\
$H(12)$ & $3360(30)$ & $6860(20)$ & $-7610(30)$ & $46(6)$ \\
$H(2)$ & $1920(30)$ & $3410(20)$ & $-4760(20)$ & $31(5)$ \\
$H(3)$ & $2160(30)$ & $3506(19)$ & $-2402(18)$ & $21(4)$ \\
$H(5)$ & $3740(30)$ & $7940(20)$ & $-1773(19)$ & $24(4)$ \\
$H(6)$ & $3630(30)$ & $7860(20)$ & $-4160(20)$ & $29(5)$ \\
$H(901)$ & $1830(30)$ & $790(30)$ & $2640(30)$ & $47(6)$ \\
$H(902)$ & $1760(40)$ & $2070(30)$ & $2400(30)$ & $53(7)$ \\
$H(911)$ & $3990(50)$ & $1280(30)$ & $-630(30)$ & $80(9)$ \\
$H(912)$ & $2820(30)$ & $570(20)$ & $-1730(30)$ & $51(7)$ \\
\hline
\end{tabular}




\section{Complex 1c}

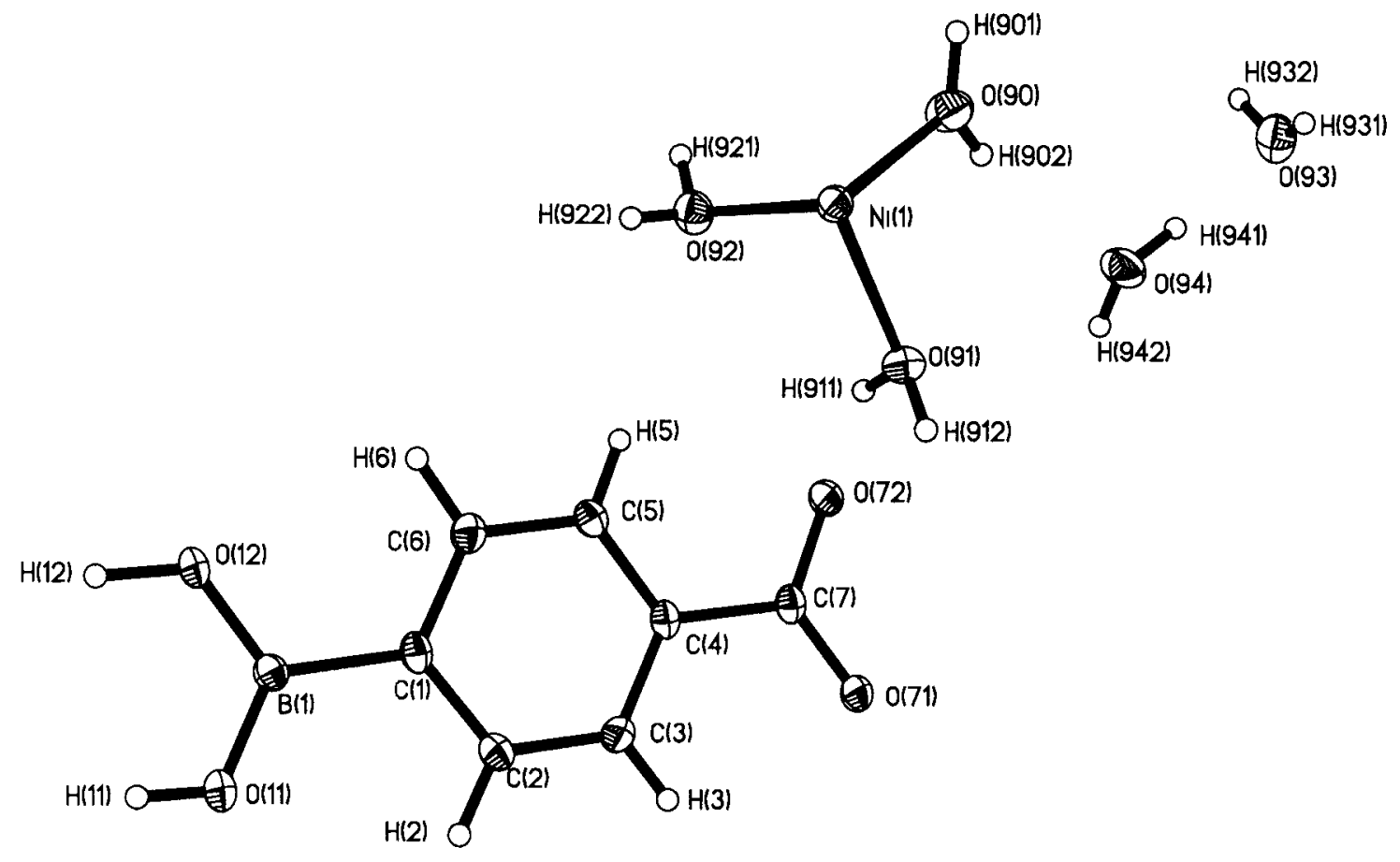

\section{Experimental Information:}

To a hot aqueous solution of $\mathrm{Ni}\left(\mathrm{NO}_{3}\right)_{2} .6 \mathrm{H}_{2} \mathrm{O}(0.290 \mathrm{~g}, 1 \mathrm{mmol})$, added a methanolic solution $(5 \mathrm{~mL})$ of sodium salt of 4-carboxyphenylboronic acid $(0.187 \mathrm{~g}, 1 \mathrm{mmol})$, with constant stirring over a period of five minutes. The reaction mixture was warmed for a while and the resulting pale blue colored solution was filtered and kept for slow evaporation at room temperature. Single crystals (blue colored) suitable for x-ray diffraction studies were obtained after one week. (Yield: 64\%). 

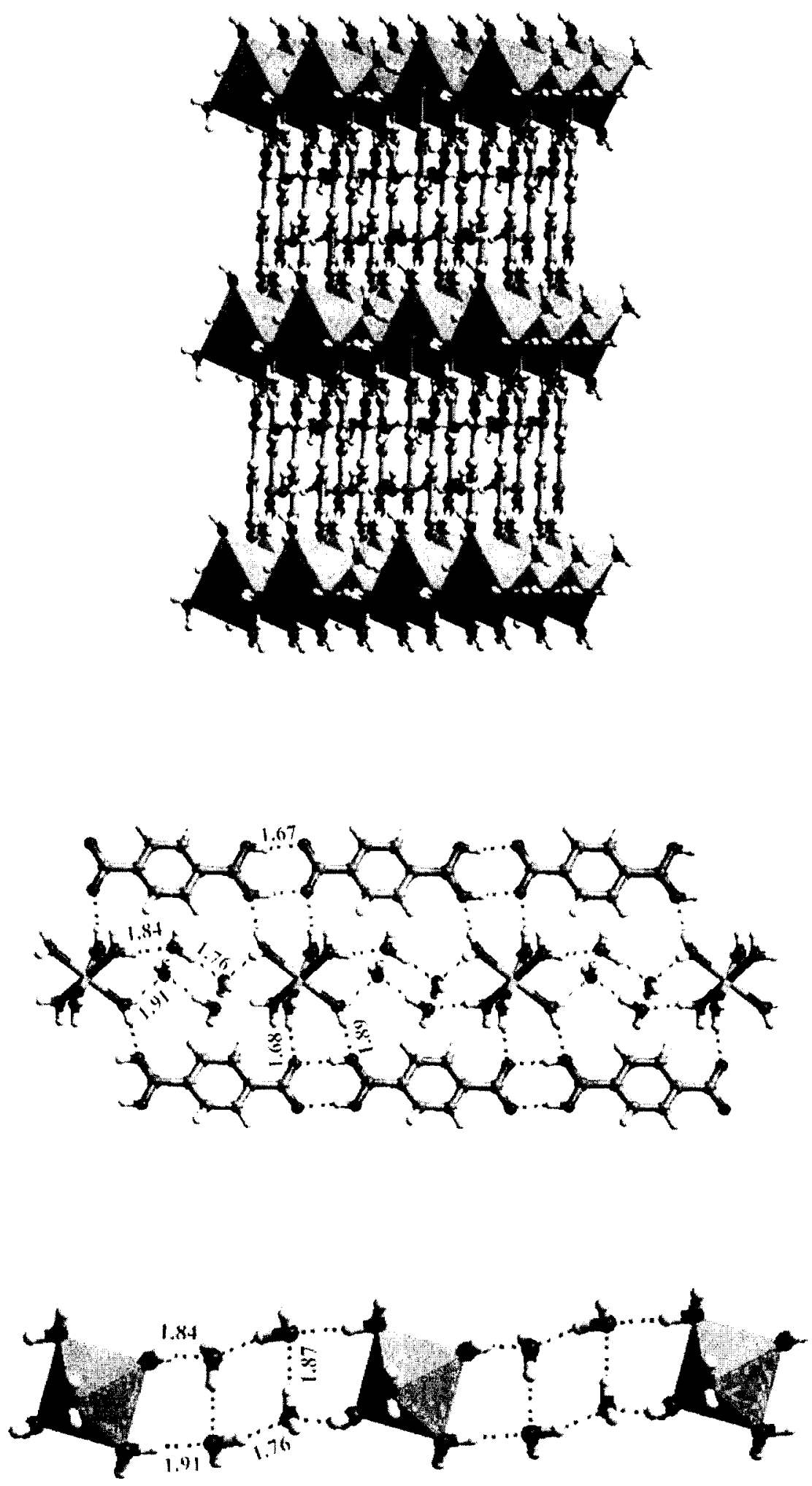


\section{Crystallographic information}

\begin{tabular}{|c|c|c|}
\hline Identification code & \multicolumn{2}{|l|}{ Ic } \\
\hline Empirical formula & \multicolumn{2}{|l|}{$\mathrm{C}_{14} \mathrm{H}_{32} \mathrm{~B}_{2} \mathrm{O}_{18} \mathrm{Ni}$} \\
\hline Formula weight & \multicolumn{2}{|l|}{568.73} \\
\hline Temperature & \multicolumn{2}{|l|}{$133(2) \mathrm{K}$} \\
\hline Wavelength & \multicolumn{2}{|l|}{$0.71073 \AA$} \\
\hline Crystal system & \multicolumn{2}{|l|}{ Triclinic } \\
\hline Space group & \multicolumn{2}{|l|}{$P \overline{1}$} \\
\hline \multirow[t]{3}{*}{ Unit cell dimensions } & $a=6.781(2) \AA$ & $\alpha=97.89(4)^{\circ}$ \\
\hline & $\mathrm{b}=9.572(3) \AA$ & $\beta=92.05(5)^{\circ}$ \\
\hline & $c=9.824(3) \AA$ & $\gamma=108.55(4)^{\circ}$ \\
\hline Volume & \multicolumn{2}{|l|}{$596.7(3) \AA^{3}$} \\
\hline Z & \multicolumn{2}{|l|}{1} \\
\hline Density (calculated) & \multicolumn{2}{|l|}{$1.583 \mathrm{Mg} / \mathrm{m}^{3}$} \\
\hline Absorption coefficient & \multicolumn{2}{|l|}{$0.897 \mathrm{~mm}^{-1}$} \\
\hline $\mathrm{F}(000)$ & \multicolumn{2}{|l|}{298} \\
\hline Crystal size & \multicolumn{2}{|c|}{$0.31 \times 0.23 \times 0.19 \mathrm{~mm}^{3}$} \\
\hline Theta range for data collection & \multicolumn{2}{|l|}{2.10 to $28.19^{\circ}$} \\
\hline Index ranges & \multicolumn{2}{|c|}{$-8 \leq \mathrm{h} \geq 6,-11 \leq \mathrm{k} \geq 12,-12 \leq \mathrm{l} \geq 12$} \\
\hline Reflections collected & \multicolumn{2}{|l|}{3532} \\
\hline Independent reflections & \multicolumn{2}{|c|}{$2557[\mathrm{R}(\mathrm{int})=0.0193]$} \\
\hline Completeness to theta $=28.19^{\circ}$ & \multicolumn{2}{|l|}{$87.6 \%$} \\
\hline Absorption correction & \multicolumn{2}{|l|}{ Empirical } \\
\hline Refinement method & \multicolumn{2}{|c|}{ Full-matrix least-squares on $\mathrm{F}^{2}$} \\
\hline Data / restraints / parameters & \multicolumn{2}{|l|}{$2557 / 0 / 224$} \\
\hline Goodness-of-fit on $\mathrm{F}^{2}$ & \multicolumn{2}{|l|}{1.016} \\
\hline Final $R$ indices $[\mathrm{I}>2 \operatorname{sigma}(\mathrm{I})]$ & \multicolumn{2}{|c|}{$\mathrm{R} 1=0.0412, w R 2=0.0978$} \\
\hline $\mathrm{R}$ indices (all data) & \multicolumn{2}{|c|}{$\mathrm{R} 1=0.0474, w \mathrm{R} 2=0.1000$} \\
\hline Largest diff. peak and hole & \multicolumn{2}{|c|}{0.749 and -0.403 e. $\AA^{-3}$} \\
\hline
\end{tabular}


Table 2. Atomic coordinates ( $\left.\times 10^{4}\right)$ and equivalent isotropic displacement parameters $\left(\AA^{2} \times 10^{3}\right)$ for 1 c. $U(e q)$ is defined as one third of the trace of the orthogonalized $U^{i j}$ tensor.

\begin{tabular}{|c|c|c|c|c|}
\hline & $\mathrm{x}$ & $y$ & $\mathrm{z}$ & $\mathrm{U}(\mathrm{eq})$ \\
\hline $\mathrm{Ni}(1)$ & 0 & 5000 & 0 & $15(1)$ \\
\hline$O(90)$ & $1403(3)$ & $6167(2)$ & $1905(2)$ & $25(1)$ \\
\hline$O(91)$ & $407(3)$ & $3181(2)$ & $635(2)$ & $20(1)$ \\
\hline$O(92)$ & $2856(3)$ & $5452(2)$ & $-806(2)$ & $21(1)$ \\
\hline$O(93)$ & $2645(4)$ & $5839(2)$ & $6423(2)$ & $27(1)$ \\
\hline$(0(94)$ & $1547(3)$ & $4253(2)$ & $3817(2)$ & $26(1)$ \\
\hline$B(1)$ & $2786(4)$ & $616(3)$ & $-6365(3)$ & $15(1)$ \\
\hline$C(1)$ & $2793(4)$ & $659(3)$ & $-4773(2)$ & $16(1)$ \\
\hline$C(2)$ & $2268(4)$ & $-663(3)$ & $-4202(2)$ & $19(1)$ \\
\hline$C(3)$ & $2340(4)$ & $-637(3)$ & $-2793(2)$ & $18(1)$ \\
\hline$C(4)$ & $2918(3)$ & $713(2)$ & $-1888(2)$ & $15(1)$ \\
\hline$C(5)$ & $3413(4)$ & $2036(3)$ & $-2435(2)$ & $18(1)$ \\
\hline$C(6)$ & $3358(4)$ & $2007(3)$ & $-3857(2)$ & $19(1)$ \\
\hline$C(7)$ & $3012(3)$ & $728(2)$ & $-350(2)$ & $16(1)$ \\
\hline$O(11)$ & $2232(3)$ & $-703(2)$ & $-7221(2)$ & $20(1)$ \\
\hline$O(12)$ & $3378(3)$ & $1946(2)$ & $-6863(2)$ & $18(1)$ \\
\hline$O(71)$ & $2435(3)$ & $-505(2)$ & $119(2)$ & $21(1)$ \\
\hline$O(72)$ & $3645(2)$ & $1999(2)$ & $452(2)$ & $17(1)$ \\
\hline
\end{tabular}

Table 3. Bond lengths $[\AA]$ and angles $\left[{ }^{\circ}\right]$ for $\mathbf{l}$.

\begin{tabular}{|c|c|c|c|}
\hline $\mathrm{Ni}(1)-\mathrm{O}(91)$ & $2.0217(18)$ & $\mathrm{O}(91)-\mathrm{Ni}(1)-\mathrm{O}(91) \# 1$ & 180.0 \\
\hline $\mathrm{Ni}(1)-\mathrm{O}(91) \# 1$ & $2.0217(18)$ & $\mathrm{O}(91)-\mathrm{Ni}(1)-\mathrm{O}(92) \# 1$ & $90.83(8)$ \\
\hline $\mathrm{Ni}(1)-\mathrm{O}(92) \# 1$ & $2.0580(19)$ & $\mathrm{O}(91) \# 1-\mathrm{Ni}(1)-\mathrm{O}(92) \# 1$ & $89.17(8)$ \\
\hline $\mathrm{Ni}(1)-\mathrm{O}(92)$ & $2.0580(19)$ & $\mathrm{O}(91)-\mathrm{Ni}(1)-\mathrm{O}(92)$ & $89.17(8)$ \\
\hline $\mathrm{Ni}(1)-\mathrm{O}(90)$ & $2.0680(19)$ & $\mathrm{O}(91) \# 1-\mathrm{Ni}(1)-\mathrm{O}(92)$ & $90.83(8)$ \\
\hline $\mathrm{Ni}(1)-\mathrm{O}(90) \# 1$ & $2.0680(19)$ & $\mathrm{O}(92) \# 1-\mathrm{Ni}(1)-\mathrm{O}(92)$ & 180.0 \\
\hline $\mathrm{B}(1)-\mathrm{O}(11)$ & $1.352(3)$ & $\mathrm{O}(91)-\mathrm{Ni}(1)-\mathrm{O}(90)$ & $86.91(8)$ \\
\hline $\mathrm{B}(1)-\mathrm{O}(12)$ & $1.371(3)$ & $\mathrm{O}(91) \# 1-\mathrm{Ni}(1)-\mathrm{O}(90)$ & $93.09(8)$ \\
\hline$B(1)-C(1)$ & $1.558(3)$ & $\mathrm{O}(92) \# 1-\mathrm{Ni}(1)-\mathrm{O}(90)$ & $89.10(8)$ \\
\hline$C(1)-C(6)$ & $1.402(3)$ & $\mathrm{O}(92)-\mathrm{Ni}(1)-\mathrm{O}(90)$ & $90.90(8)$ \\
\hline$C(1)-C(2)$ & $1.402(3)$ & $\mathrm{O}(91)-\mathrm{Ni}(1)-\mathrm{O}(90) \# \mathrm{I}$ & $93.09(8)$ \\
\hline$C(2)-C(3)$ & $1.381(3)$ & $\mathrm{O}(91) \# 1-\mathrm{Ni}(1)-\mathrm{O}(90) \# 1$ & $86.91(8)$ \\
\hline$C(3)-C(4)$ & $1.397(3)$ & $\mathrm{O}(92) \# 1-\mathrm{Ni}(1)-\mathrm{O}(90) \# 1$ & $90.90(8)$ \\
\hline$C(4)-C(5)$ & $1.391(3)$ & $\mathrm{O}(92)-\mathrm{Ni}(1)-\mathrm{O}(90) \# \mathrm{I}$ & $89.10(8)$ \\
\hline$C(4)-C(7)$ & $1.507(3)$ & $\mathrm{O}(90)-\mathrm{Ni}(1)-\mathrm{O}(90) \# 1$ & $180.00(11)$ \\
\hline$C(5)-C(6)$ & $1.392(3)$ & $\mathrm{O}(11)-\mathrm{B}(1)-\mathrm{O}(12)$ & $121.4(2)$ \\
\hline$C(7)-O(71)$ & $1.276(3)$ & $\mathrm{O}(11)-\mathrm{B}(1)-\mathrm{C}(1)$ & $120.4(2)$ \\
\hline \multirow[t]{14}{*}{$C(7)-O(72)$} & $1.291(3)$ & $O(12)-B(1)-C(1)$ & $118.2(2)$ \\
\hline & & $C(6)-C(1)-C(2)$ & $117.4(2)$ \\
\hline & & $\mathrm{C}(6)-\mathrm{C}(1)-\mathrm{B}(1)$ & $121.9(2)$ \\
\hline & & $C(2)-C(1)-B(1)$ & $120.8(2)$ \\
\hline & & $C(3)-C(2)-C(1)$ & $121.3(2)$ \\
\hline & & $C(2)-C(3)-C(4)$ & $120.9(2)$ \\
\hline & & $\mathrm{C}(5)-\mathrm{C}(4)-\mathrm{C}(3)$ & $118.7(2)$ \\
\hline & & $C(5)-C(4)-C(7)$ & $121.0(2)$ \\
\hline & & $C(3)-C(4)-C(7)$ & $120.4(2)$ \\
\hline & & $C(4)-C(5)-C(6)$ & $120.4(2)$ \\
\hline & & $C(5)-C(6)-C(1)$ & $121.4(2)$ \\
\hline & & $\mathrm{O}(71)-\mathrm{C}(7)-\mathrm{O}(72)$ & $122.0(2)$ \\
\hline & & $O(71)-C(7)-C(4)$ & $119.39(19)$ \\
\hline & & $O(72)-C(7)-C(4)$ & $118.6(2)$ \\
\hline
\end{tabular}


Table 4. Anisotropic displacement parameters $\left(\AA^{2} \times 10^{3}\right)$ for 1 c. The anisotropic displacement factor exponent takes the form: $-2 \pi^{2}\left[h^{2} a^{* 2} U^{11}+\ldots+2 h k a^{*} b^{*} U^{12}\right]$

\begin{tabular}{lllllll}
\hline & $U^{11}$ & $U^{22}$ & $U^{33}$ & $U^{23}$ & $U^{13}$ & $U^{12}$ \\
\hline $\mathrm{Ni}(1)$ & $17(1)$ & $14(1)$ & $13(1)$ & $1(1)$ & $0(1)$ & $3(1)$ \\
$O(90)$ & $35(1)$ & $18(1)$ & $18(1)$ & $2(1)$ & $-5(1)$ & $5(1)$ \\
$O(91)$ & $20(1)$ & $16(1)$ & $22(1)$ & $3(1)$ & $1(1)$ & $5(1)$ \\
$O(92)$ & $20(1)$ & $22(1)$ & $17(1)$ & $1(1)$ & $0(1)$ & $3(1)$ \\
$O(93)$ & $25(1)$ & $28(1)$ & $20(1)$ & $-1(1)$ & $1(1)$ & $0(1)$ \\
$O(94)$ & $38(1)$ & $23(1)$ & $17(1)$ & $-2(1)$ & $-1(1)$ & $13(1)$ \\
$B(1)$ & $15(1)$ & $16(1)$ & $13(1)$ & $2(1)$ & $-1(1)$ & $5(1)$ \\
$C(1)$ & $15(1)$ & $21(1)$ & $11(1)$ & $2(1)$ & $0(1)$ & $6(1)$ \\
$C(2)$ & $22(1)$ & $18(1)$ & $13(1)$ & $0(1)$ & $1(1)$ & $4(1)$ \\
$C(3)$ & $22(1)$ & $16(1)$ & $15(1)$ & $4(1)$ & $2(1)$ & $4(1)$ \\
$C(4)$ & $14(1)$ & $20(1)$ & $11(1)$ & $3(1)$ & $2(1)$ & $5(1)$ \\
$C(5)$ & $23(1)$ & $17(1)$ & $12(1)$ & $0(1)$ & $0(1)$ & $5(1)$ \\
$C(6)$ & $23(1)$ & $19(1)$ & $14(1)$ & $4(1)$ & $0(1)$ & $6(1)$ \\
$C(7)$ & $15(1)$ & $20(1)$ & $12(1)$ & $2(1)$ & $1(1)$ & $5(1)$ \\
$O(1)$ & $26(1)$ & $20(1)$ & $12(1)$ & $3(1)$ & $1(1)$ & $6(1)$ \\
$O(12)$ & $26(1)$ & $19(1)$ & $10(1)$ & $1(1)$ & $1(1)$ & $6(1)$ \\
$O(71)$ & $29(1)$ & $19(1)$ & $11(1)$ & $1(1)$ & $1(1)$ & $3(1)$ \\
$O(72)$ & $20(1)$ & $16(1)$ & $13(1)$ & $0(1)$ & $-1(1)$ & $3(1)$ \\
\hline
\end{tabular}

Table 5. Hydrogen coordinates $\left(\times 10^{4}\right)$ and isotropic displacement parameters $\left(\AA^{2} \times 10^{3}\right)$ for $\mathbf{1 c}$.

\begin{tabular}{|c|c|c|c|c|}
\hline & $x$ & $y$ & $z$ & $\mathrm{U}(\mathrm{eq})$ \\
\hline $\mathrm{H}(912)$ & $-550(50)$ & $2320(30)$ & $480(30)$ & $22(7)$ \\
\hline $\mathrm{H}(902)$ & $1770(50)$ & $5720(30)$ & $2340(30)$ & $27(9)$ \\
\hline $\mathrm{H}(901)$ & $1680(40)$ & $7070(40)$ & $2210(30)$ & $30(8)$ \\
\hline $\mathrm{H}(922)$ & $2770(60)$ & $5420(40)$ & $-1550(40)$ & $47(12)$ \\
\hline $\mathrm{H}(2)$ & $1860(40)$ & $-1580(30)$ & $-4850(30)$ & $25(7)$ \\
\hline $\mathrm{H}(3)$ & $2000(40)$ & $-1520(30)$ & $-2430(30)$ & $23(7)$ \\
\hline$H(5)$ & $3840(40)$ & $2950(30)$ & $-1850(30)$ & $19(7)$ \\
\hline$H(6)$ & $3810(50)$ & $2950(30)$ & $-4190(30)$ & $32(8)$ \\
\hline$H(11)$ & $2310(60)$ & $-650(40)$ & $-8070(40)$ & $70(13)$ \\
\hline $\mathrm{H}(12)$ & $3550(60)$ & $1980(40)$ & $-7870(40)$ & $65(12)$ \\
\hline $\mathrm{H}(911)$ & $1460(50)$ & $3030(30)$ & $450(40)$ & $38(10)$ \\
\hline $\mathrm{H}(921)$ & $3860(70)$ & $6270(50)$ & $-590(40)$ & $66(12)$ \\
\hline $\mathrm{H}(942)$ & $1860(50)$ & $3600(40)$ & $3550(40)$ & $38(10)$ \\
\hline $\mathrm{H}(932)$ & $3620(60)$ & $6500(40)$ & $6400(40)$ & $40(11)$ \\
\hline $\mathrm{H}(941)$ & $2020(70)$ & $4750(50)$ & $4720(60)$ & $108(18)$ \\
\hline $\mathrm{H}(931)$ & $1910(120)$ & $5880(80)$ & $6490(80)$ & $160(40)$ \\
\hline
\end{tabular}




\section{Complex 1d:}

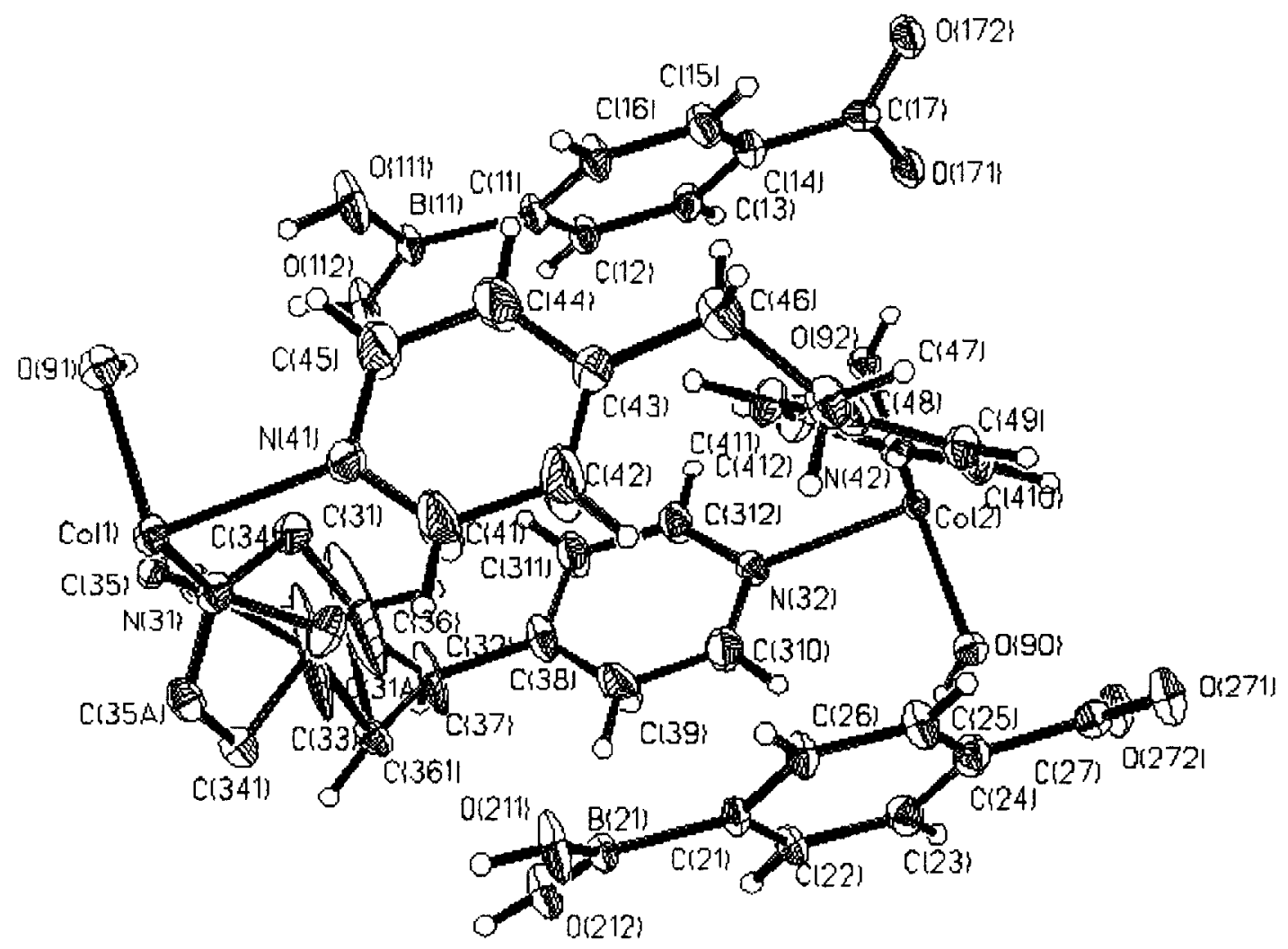

\section{Experimental Information:}

To a hot aqueous solution of $\mathrm{Co}\left(\mathrm{NO}_{3}\right)_{2} .6 \mathrm{H}_{2} \mathrm{O}(0.291 \mathrm{~g}, 1 \mathrm{mmol})$ added a methanolic solution $(5 \mathrm{~mL})$ of sodium salt of 4-carboxyphenylboronic acid $(0.187 \mathrm{gm}, 1 \mathrm{mmol})$, with constant stirring over a period of five minutes. To this mixture 1,2 -bis-4-pyridyl ethane $(0.184 \mathrm{~g}, 1 \mathrm{mmol})$ in methanol $(5 \mathrm{~mL})$ was added dropwise. This reaction mixture was warmed for about 20 minutes and the resulting pink solution was filtered and kept for slow evaporation at room temperature. Pink colored block-like crystals suitable for x-ray diffraction studies were obtained over a period of one week. (Yield: $56 \%$ ). 


\section{Crystallographic information}

Table 1. Crystal data and structure refinement for $\mathbf{1 d}$.

Identification code

Empirical formula

Formula weight

Temperature

Wavelength

Crystal system

Space group

Unit cell dimensions

Volume

Z

Density (calculated)

Absorption coefficient

$F(000)$

Crystal size

Theta range for data collection

Index ranges

Reflections collected

Independent reflections

Completeness to theta $=28.25^{\circ}$

Absorption correction

Refinement method

Data / restraints / parameters

Goodness-of-fit on $\mathrm{F}^{2}$

Final $R$ indices [ $>2 \operatorname{sigma}(I)]$

$\mathrm{R}$ indices (all data)

Largest diff. peak and hole
$1 \mathrm{~d}$

$\mathrm{C}_{38} \mathrm{H}_{33} \mathrm{~B}_{2} \mathrm{~N}_{4} \mathrm{O}_{10} \mathrm{Co}$

786.23

133(2) K

$0.71073 \AA$

Monoclinic

$\mathrm{C} 2 / \mathrm{c}$

$\mathrm{a}=19.478(5) \AA$

$\alpha=90^{\circ}$.

$\mathrm{b}=16.672(4) \AA$

$\beta=93.23(5)^{\circ}$.

$c=22.781(6) \AA$

$\gamma=90^{\circ}$.

8

$1.414 \mathrm{Mg} / \mathrm{m}^{3}$

$0.529 \mathrm{~mm}^{-1}$

3248

$0.32 \times 0.22 \times 0.20 \mathrm{~mm}^{3}$

1.61 to $28.25^{\circ}$.

$-25 \leq h \geq 23,-12 \leq k \geq 21,-29 \leq 1 \geq 29$

21571

$8363[\mathrm{R}($ int $)=0.0355]$

$91.5 \%$

Empirical

Full-matrix least-squares on $\mathrm{F}^{2}$

$8363 / 0 / 539$

0.954

$R 1=0.0909, w R 2=0.2383$

$\mathrm{R} 1=0.1328, w R 2=0.2725$

4.577 and -0.734 e. $\AA^{-3}$ 
Table 2. Atomic coordinates ( $\left.\times 10^{4}\right)$ and equivalent isotropic displacement parameters $\left(\AA^{2} \times 10^{3}\right)$ for $\mathbf{1 d}$. U(eq) is defined as one third of the trace of the orthogonalized $U^{i j}$ tensor.

\begin{tabular}{|c|c|c|c|c|}
\hline & $x$ & $y$ & $z$ & $\mathrm{U}(\mathrm{eq})$ \\
\hline $\mathrm{Co}(2)$ & 5000 & $54(1)$ & 2500 & $13(1)$ \\
\hline $\operatorname{Co}(1)$ & 0 & 0 & 0 & $16(1)$ \\
\hline$O(92)$ & 5000 & $-1163(2)$ & 2500 & $18(1)$ \\
\hline$O(91)$ & $-30(1)$ & $-1229(2)$ & $-8(1)$ & $19(1)$ \\
\hline$O(90)$ & 5000 & $1286(2)$ & 2500 & $18(1)$ \\
\hline$O(272)$ & $1798(1)$ & $2373(2)$ & $1774(1)$ & $28(1)$ \\
\hline$O(112)$ & $5456(1)$ & $-2211(2)$ & $1714(1)$ & $28(1)$ \\
\hline$N(31)$ & $332(2)$ & $14(2)$ & $932(2)$ & $20(1)$ \\
\hline$N(42)$ & $4678(2)$ & $58(2)$ & $1565(1)$ & $16(1)$ \\
\hline$O(172)$ & $1806(1)$ & $-2286(2)$ & $1770(1)$ & $33(1)$ \\
\hline$O(111)$ & $5396(1)$ & $-2648(2)$ & $797(1)$ & $29(1)$ \\
\hline$O(2 \mid 2)$ & $5450(1)$ & $2349(2)$ & $1731(1)$ & $26(1)$ \\
\hline$O(211)$ & $5397(1)$ & $2698(2)$ & $796(1)$ & $27(1)$ \\
\hline$O(271)$ & $1739(1)$ & $2487(2)$ & $720(1)$ & $33(1)$ \\
\hline$N(41)$ & $1116(2)$ & $-29(2)$ & $-188(2)$ & $21(1)$ \\
\hline$O(171)$ & $1743(1)$ & $-2452(2)$ & $720(1)$ & $38(1)$ \\
\hline$B(11)$ & $5124(2)$ & $-2420(2)$ & $1250(2)$ & $14(1)$ \\
\hline$C(13)$ & $3305(2)$ & $-2406(2)$ & $1773(2)$ & $19(1)$ \\
\hline$N(32)$ & $3890(2)$ & $83(2)$ & $2704(1)$ & $17(1)$ \\
\hline$C(11)$ & $4354(2)$ & $-2395(2)$ & $1248(2)$ & $24(1)$ \\
\hline$C(23)$ & $3299(2)$ & $2573(2)$ & $1775(2)$ & $16(1)$ \\
\hline$B(21)$ & $5123(2)$ & $2533(2)$ & $1262(2)$ & $12(1)$ \\
\hline$C(24)$ & $2906(2)$ & $2452(2)$ & $1253(2)$ & $15(1)$ \\
\hline$C(22 A)$ & $4015(2)$ & $2611(2)$ & $1781(2)$ & $2 !(1)$ \\
\hline$C(25)$ & $3245(2)$ & $2379(2)$ & $732(2)$ & $20(1)$ \\
\hline$c(12)$ & $4020(2)$ & $-2443(2)$ & $1775(2)$ & $22(1)$ \\
\hline$C(4 \mid 1)$ & $3782(2)$ & $-172(2)$ & $819(2)$ & $24(1)$ \\
\hline$C(3 \mid 2)$ & $3563(2)$ & $-545(2)$ & $2915(2)$ & $28(1)$ \\
\hline$C(21)$ & $4346(2)$ & $2526(2)$ & $1257(2)$ & $23(1)$ \\
\hline$C(410)$ & $4986(2)$ & $515(2)$ & $1176(2)$ & $21(1)$ \\
\hline$C(26)$ & $3954(2)$ & $2417(2)$ & $732(2)$ & $23(1)$ \\
\hline$C(48)$ & $4093(2)$ & $308(2)$ & $418(2)$ & $22(1)$ \\
\hline$C(15)$ & $3248(2)$ & $-2297(3)$ & $724(2)$ & $23(1)$ \\
\hline$C(27)$ & $2098(2)$ & $2426(3)$ & $1248(2)$ & $26(1)$ \\
\hline$C(49)$ & $4720(2)$ & $653(2)$ & $612(2)$ & $21(1)$ \\
\hline$C(46)$ & $3297(2)$ & $-148(3)$ & $-468(2)$ & $28(1)$ \\
\hline$C(14)$ & $2912(2)$ & $-2340(2)$ & $1246(2)$ & $19(1)$ \\
\hline$C(412)$ & $4086(2)$ & $-287(2)$ & $1374(2)$ & $24(1)$ \\
\hline$C(43)$ & $2542(2)$ & $-88(2)$ & $-338(2)$ & $21(1)$ \\
\hline$C(39)$ & $2792(2)$ & $745(3)$ & $2628(2)$ & $40(1)$ \\
\hline$C(17)$ & $2103(2)$ & $-2354(3)$ & $1246(2)$ & $29(1)$ \\
\hline$C(16)$ & $3959(2)$ & $-2324(3)$ & $721(2)$ & $26(1)$ \\
\hline$C(311)$ & $2858(2)$ & $-563(3)$ & $2981(2)$ & $32(1)$ \\
\hline$C(38)$ & $2459(2)$ & 95(3) & $2845(2)$ & $23(1)$ \\
\hline$C(47)$ & $3788(2)$ & $492(3)$ & $-190(2)$ & $26(1)$ \\
\hline$C(41)$ & $1494(3)$ & $603(3)$ & $-241(4)$ & $103(3)$ \\
\hline$C(310)$ & $3501(2)$ & $716(3)$ & $2563(2)$ & $32(1)$ \\
\hline$C(37)$ & $1703(2)$ & $104(4)$ & $2963(2)$ & $48(2)$ \\
\hline$C(44)$ & $2160(3)$ & $-731(3)$ & $-311(4)$ & $93(3)$ \\
\hline$C(45)$ & $1460(3)$ & $-685(3)$ & $-236(4)$ & $94(3)$ \\
\hline$C(42)$ & $2199(3)$ & $588(3)$ & $-314(4)$ & $107(4)$ \\
\hline$C(35 A)$ & $26(4)$ & $528(5)$ & $1303(3)$ & $22(2)$ \\
\hline$C(341)$ & $295(4)$ & $709(5)$ & $1860(3)$ & $20(2)$ \\
\hline$C(33)$ & $908(3)$ & $63(6)$ & $2079(2)$ & $125(5)$ \\
\hline$C(34)$ & $315(4)$ & $-596(5)$ & $1878(3)$ & $20(2)$ \\
\hline$(435)$ & $43(4)$ & $-463(5)$ & $1309(3)$ & $16(2)$ \\
\hline$C(3 \mid A)$ & $912(4)$ & $412(5)$ & $1119(3)$ & $27(2)$ \\
\hline$C(3 I)$ & $888(4)$ & $-378(5)$ & $1152(3)$ & $22(2)$ \\
\hline$C(361)$ & $1198(4)$ & $554(5)$ & $2680(3)$ & $22(2)$ \\
\hline$C(36)$ & $1238(4)$ & $-409(5)$ & $2674(3)$ & $19(2)$ \\
\hline
\end{tabular}


Table 3. Bond lengths $[\AA]$ and angles $\left[{ }^{\circ}\right]$ for $\mathbf{1 d}$.

Table 4. Anisotropic displacement parameters $\left(\AA^{2} \times 10^{3}\right)$ for $1 \mathbf{d}$. The anisotropic displacement factor exponent takes the form: $-2 \pi^{2}\left[h^{2} a^{* 2} U^{11}+\ldots+2 h k a^{*} b^{*} U^{12}\right]$

\begin{tabular}{|c|c|c|c|c|c|c|}
\hline & $U^{\prime \prime}$ & $\mathrm{U}^{22}$ & $U^{33}$ & $\mathrm{U}^{23}$ & $\mathrm{U}^{13}$ & $U^{12}$ \\
\hline $\operatorname{Co}(2)$ & $11(1)$ & $16(1)$ & $12(1)$ & 0 & $-1(1)$ & 0 \\
\hline $\operatorname{Co}(1)$ & $15(1)$ & $18(1)$ & $15(1)$ & $0(1)$ & $-5(1)$ & $0(1)$ \\
\hline$O(92)$ & $18(2)$ & $18(2)$ & $17(2)$ & 0 & $4(1)$ & 0 \\
\hline$O(91)$ & $21(1)$ & $22(1)$ & $14(1)$ & $2(1)$ & $-4(1)$ & I(1) \\
\hline$O(90)$ & $19(2)$ & $20(2)$ & $14(2)$ & 0 & $2(1)$ & 0 \\
\hline$O(272)$ & $15(1)$ & $51(2)$ & $19(1)$ & $0(1)$ & $4(1)$ & $2(1)$ \\
\hline$O(112)$ & $14(1)$ & $38(2)$ & $30(2)$ & $-11(1)$ & 1(1) & $O(1)$ \\
\hline$N(31)$ & $20(2)$ & $22(2)$ & $18(2)$ & $0(1)$ & $-6(1)$ & $-1(1)$ \\
\hline$N(42)$ & $15(2)$ & $18(2)$ & $15(2)$ & $0(1)$ & $-1(1)$ & $1(1)$ \\
\hline$O(172)$ & $12(1)$ & $68(2)$ & $18(2)$ & $-2(1)$ & $3(1)$ & $-3(1)$ \\
\hline$O(111)$ & $15(1)$ & $43(2)$ & $30(2)$ & $-12(1)$ & $2(1)$ & $-2(1)$ \\
\hline $\mathrm{O}(212)$ & $14(1)$ & $38(2)$ & $26(2)$ & $6(1)$ & $0(1)$ & $-2(1)$ \\
\hline$O(2 \mid 1)$ & $15(1)$ & $42(2)$ & $25(2)$ & $10(1)$ & $2(1)$ & $1(1)$ \\
\hline$O(271)$ & $9(1)$ & $73(2)$ & $18(1)$ & $4(1)$ & $0(1)$ & $-2(1)$ \\
\hline$N(41)$ & $18(2)$ & $21(2)$ & $23(2)$ & $0(1)$ & $-3(1)$ & $1(1)$ \\
\hline$O(171)$ & $10(1)$ & $84(3)$ & $19(2)$ & $-3(2)$ & $0(1)$ & $2(2)$ \\
\hline$B(11)$ & $14(2)$ & $15(2)$ & $13(2)$ & $-6(2)$ & $1(2)$ & $-1(2)$ \\
\hline$C(13)$ & $14(2)$ & $28(2)$ & $16(2)$ & $-2(2)$ & $2(2)$ & $-4(2)$ \\
\hline$N(32)$ & $13(2)$ & $23(2)$ & $15(2)$ & $-1(1)$ & $-4(1)$ & $3(1)$ \\
\hline$C(11)$ & $14(2)$ & $26(2)$ & $31(2)$ & $-2(2)$ & $3(2)$ & $0(2)$ \\
\hline$C(23)$ & $12(2)$ & $23(2)$ & $12(2)$ & $2(1)$ & $1(1)$ & $3(1)$ \\
\hline$B(21)$ & $13(2)$ & $12(2)$ & $12(2)$ & $2(2)$ & $O(2)$ & $0(2)$ \\
\hline$C(24)$ & $11(2)$ & $17(2)$ & $18(2)$ & $1(1)$ & $0(1)$ & $-1(1)$ \\
\hline$C(22 A)$ & $21(2)$ & $23(2)$ & $19(2)$ & $0(2)$ & $-1(2)$ & $1(2)$ \\
\hline$C(25)$ & $12(2)$ & $31(2)$ & $16(2)$ & $-1(2)$ & $0(1)$ & $-2(2)$ \\
\hline$C(12)$ & $18(2)$ & $28(2)$ & $21(2)$ & $-2(2)$ & $0(2)$ & $-4(2)$ \\
\hline$C(411)$ & $22(2)$ & $28(2)$ & $21(2)$ & $-2(2)$ & $-4(2)$ & $-7(2)$ \\
\hline$C(312)$ & $20(2)$ & $22(2)$ & $42(3)$ & $10(2)$ & $10(2)$ & $2(2)$ \\
\hline$C(21)$ & $17(2)$ & $24(2)$ & $29(2)$ & $1(2)$ & $1(2)$ & $-1(2)$ \\
\hline$C(410)$ & $16(2)$ & $26(2)$ & $20(2)$ & $-1(2)$ & $-1(2)$ & $-2(2)$ \\
\hline$C(26)$ & $17(2)$ & $33(2)$ & $20(2)$ & $1(2)$ & $3(2)$ & $0(2)$ \\
\hline$C(48)$ & $16(2)$ & $26(2)$ & $23(2)$ & $-4(2)$ & $0(2)$ & $2(2)$ \\
\hline$C(15)$ & $11(2)$ & $41(2)$ & $16(2)$ & $-2(2)$ & $-2(2)$ & $1(2)$ \\
\hline$C(27)$ & $20(2)$ & $31(2)$ & $26(2)$ & $-1(2)$ & $2(2)$ & $1(2)$ \\
\hline$C(49)$ & $20(2)$ & $24(2)$ & $19(2)$ & $1(2)$ & $2(2)$ & $-3(2)$ \\
\hline$C(46)$ & $2 \mathrm{l}(2)$ & $44(3)$ & $19(2)$ & $-3(2)$ & $-4(2)$ & $5(2)$ \\
\hline$C(14)$ & $11(2)$ & $29(2)$ & $16(2)$ & $-2(2)$ & $2(2)$ & $-1(2)$ \\
\hline$C(412)$ & $22(2)$ & $27(2)$ & $22(2)$ & $3(2)$ & $-3(2)$ & $-5(2)$ \\
\hline$C(43)$ & $17(2)$ & $27(2)$ & $17(2)$ & $-2(2)$ & $-7(2)$ & $0(2)$ \\
\hline$C(39)$ & $22(2)$ & $42(3)$ & $55(3)$ & $11(2)$ & $4(2)$ & $16(2)$ \\
\hline$C(17)$ & $20(2)$ & $42(3)$ & $27(2)$ & $0(2)$ & $3(2)$ & $-1(2)$ \\
\hline$C(16)$ & $19(2)$ & $38(2)$ & $22(2)$ & $-4(2)$ & $4(2)$ & $0(2)$ \\
\hline$C(311)$ & $19(2)$ & $33(2)$ & $46(3)$ & $8(2)$ & $11(2)$ & $-I(2)$ \\
\hline$C(38)$ & $12(2)$ & $45(3)$ & $11(2)$ & $-3(2)$ & $-3(1)$ & $1(2)$ \\
\hline$C(47)$ & $20(2)$ & $37(2)$ & $21(2)$ & $2(2)$ & $-1(2)$ & $2(2)$ \\
\hline$C(41)$ & $24(3)$ & $21(3)$ & $268(11)$ & $16(4)$ & $37(4)$ & $9(2)$ \\
\hline$C(310)$ & $22(2)$ & $27(2)$ & $48(3)$ & $7(2)$ & $5(2)$ & $1(2)$ \\
\hline$C(37)$ & $9(2)$ & $119(5)$ & $16(2)$ & $-4(2)$ & $-3(2)$ & $6(2)$ \\
\hline$C(44)$ & $33(3)$ & $23(3)$ & $225(9)$ & $-23(4)$ & $39(4)$ & $1(2)$ \\
\hline$C(45)$ & $32(3)$ & $20(3)$ & $234(10)$ & $-19(4)$ & $43(4)$ & $-8(2)$ \\
\hline$C(42)$ & $26(3)$ & $26(3)$ & $272(11)$ & $16(5)$ & $30(5)$ & $3(2)$ \\
\hline
\end{tabular}




\begin{tabular}{llccccc}
$C(35 A)$ & $18(4)$ & $26(4)$ & $22(4)$ & $5(3)$ & $-1(3)$ & $6(3)$ \\
$C(341)$ & $19(4)$ & $26(4)$ & $14(4)$ & $3(3)$ & $1(3)$ & $4(3)$ \\
$C(33)$ & $10(3)$ & $353(15)$ & $11(3)$ & $7(4)$ & $-3(2)$ & $-13(4)$ \\
$C(34)$ & $15(4)$ & $26(4)$ & $18(4)$ & $-3(3)$ & $-1(3)$ & $-2(3)$ \\
$C(35)$ & $16(4)$ & $19(4)$ & $14(4)$ & $-4(3)$ & $2(3)$ & $-4(3)$ \\
$C(31 A)$ & $25(4)$ & $31(5)$ & $24(4)$ & $1(3)$ & $-6(3)$ & $-10(4)$ \\
$C(31)$ & $20(4)$ & $24(4)$ & $22(4)$ & $-3(3)$ & $-10(3)$ & $2(3)$ \\
$C(361)$ & $21(4)$ & $33(5)$ & $13(4)$ & $-8(3)$ & $2(3)$ & $8(3)$ \\
$C(36)$ & $15(4)$ & $26(4)$ & $15(4)$ & $5(3)$ & $2(3)$ & $-7(3)$ \\
$C(32)$ & $19(3)$ & $358(16)$ & $23(3)$ & $-32(5)$ & $-7(3)$ & $21(5)$ \\
\hline
\end{tabular}

Table 4. Hydrogen coordinates ( $\left.\times 10^{4}\right)$ and isotropic displacement parameters $\left(\AA^{2} \times 10^{3}\right)$ for $1 \mathbf{d}$.

\begin{tabular}{|c|c|c|c|c|}
\hline & $\mathrm{x}$ & $\mathrm{y}$ & $z$ & $U(e q)$ \\
\hline$\overline{\mathrm{H}(921)}$ & 5172 & -1327 & 2202 & 26 \\
\hline$H(911)$ & 141 & -1402 & 305 & 29 \\
\hline $\mathrm{H}(901)$ & 4826 & 1450 & 2797 & 26 \\
\hline $\mathrm{H}(272)$ & 1393 & 2505 & 1731 & 42 \\
\hline$H(172)$ & 1397 & -2404 & 1727 & 49 \\
\hline$H(271)$ & 1327 & 2441 & 768 & 50 \\
\hline$H(171)$ & 1331 & -2400 & 767 & 57 \\
\hline$H(13)$ & 3089 & -2426 & 2127 & 23 \\
\hline $\mathrm{H}(23)$ & 3081 & 2629 & 2126 & 19 \\
\hline $\mathrm{H}(22)$ & 4271 & 2692 & 2133 & 25 \\
\hline $\mathrm{H}(25)$ & 2990 & 2303 & 379 & 23 \\
\hline$H(12)$ & 4277 & -2501 & 2129 & 27 \\
\hline $\mathrm{H}(411)$ & 3366 & -420 & 714 & 29 \\
\hline $\mathrm{H}(312)$ & 3820 & -997 & 3024 & 34 \\
\hline $\mathrm{H}(410)$ & 5404 & 753 & 1292 & 25 \\
\hline$H(26)$ & 4170 & 2370 & 380 & 28 \\
\hline$H(15)$ & 2991 & -2249 & 369 & 27 \\
\hline $\mathrm{H}(49)$ & 4958 & 976 & 359 & 25 \\
\hline$H(462)$ & 3329 & -127 & -891 & 34 \\
\hline$H(461)$ & 3462 & -671 & -339 & 34 \\
\hline$H(412)$ & 3868 & -624 & 1630 & 29 \\
\hline $\mathrm{H}(39)$ & 2546 & 1206 & 2523 & 48 \\
\hline$H(16)$ & 4174 & -2296 & 367 & 31 \\
\hline $\mathrm{H}(311)$ & 2656 & -1026 & 3119 & 39 \\
\hline $\mathrm{H}(472)$ & 4162 & 571 & -448 & 32 \\
\hline $\mathrm{H}(471)$ & 3539 & 995 & -174 & 32 \\
\hline$H(41)$ & 1280 & 1101 & -229 & 124 \\
\hline $\mathrm{H}(310)$ & 3712 & 1166 & 2412 & 39 \\
\hline $\mathrm{H}(37)$ & 1642 & 96 & 3387 & 58 \\
\hline$H(44)$ & 2364 & -1233 & -343 & 111 \\
\hline$H(45)$ & 1218 & -1165 & -220 & 113 \\
\hline $\mathrm{H}(42)$ & 2433 & 1070 & -346 & 128 \\
\hline $\mathrm{H}(361)$ & 855 & 801 & 2922 & 26 \\
\hline$H(36)$ & 919 & -702 & 2914 & 23 \\
\hline $\mathrm{H}(32)$ & 1697 & 7 & 1765 & 160 \\
\hline
\end{tabular}


Hydrogen Bonds Table:

\begin{tabular}{|c|c|c|c|c|}
\hline \multirow[b]{2}{*}{ Complexes } & \multicolumn{4}{|c|}{ Hydrogen bonds } \\
\hline & O-H $\cdots O$ & $\mathrm{O}-\mathrm{H}^{\cdots} \mathrm{O}^{-}$ & $\mathrm{C}-\mathrm{H}^{\cdots} \mathrm{O} \mathrm{O}$ & $\mathrm{C}-\mathrm{H}^{\cdots} \mathrm{O}^{-}$ \\
\hline $1 \mathbf{a}$ & $\begin{array}{lll}1.75 & 2.72 & 166 \\
1.82 & 2.79 & 166 \\
1.87 & 2.85 & 177 \\
1.88 & 2.82 & 161 \\
1.89 & 2.83 & 157 \\
1.91 & 2.84 & 157 \\
1.91 & 2.88 & 168\end{array}$ & $\begin{array}{l}1.672 .66178 \\
1.682 .66174\end{array}$ & $\begin{array}{llll}2.62 & 3.58 & 146 \\
2.65 & 3.56 & 140 \\
2.94 & 3.78 & 135\end{array}$ & \\
\hline $1 b$ & $\begin{array}{llll}1.75 & 2.71 & 165 \\
1.80 & 2.78 & 171 \\
1.89 & 2.87 & 171 \\
1.90 & 2.86 & 164 \\
1.92 & 2.83 & 153 \\
1.94 & 2.85 & 153 \\
1.94 & 2.86 & 154\end{array}$ & $\begin{array}{l}1.672 .65178 \\
1.682 .66 \quad 170\end{array}$ & & \\
\hline 1c & $\begin{array}{lll}1.76 & 2.74 & 171 \\
1.84 & 2.80 & 165 \\
1.87 & 2.82 & 160 \\
1.88 & 2.83 & 159 \\
1.89 & 2.87 & 174 \\
1.91 & 2.82 & 153 \\
1.91 & 2.87 & 165\end{array}$ & $\begin{array}{l}1.672 .65 \quad 178 \\
1.682 .66 \quad 170\end{array}$ & $\begin{array}{c}2.633 .56144 \\
2.643 .58145 \\
2.993 .77129\end{array}$ & \\
\hline $1 d$ & 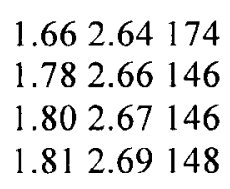 & 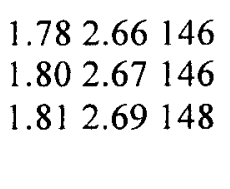 & $\begin{array}{lll}2.51 & 3.32 & 130 \\
2.57 & 3.56 & 152\end{array}$ & $\begin{array}{l}2.373 .45178 \\
2.393 .35146 \\
2.763 .79160\end{array}$ \\
\hline
\end{tabular}

$\mathrm{H}^{*} \mathrm{~A}$ distances, D A distances and angles of different type of hydrogen bonds observed in the crystal structure of metal complexes of boronic acids. $(A=A c c e p t o r, D=D o n o r)$ 